\title{
Mitochondrial protein import under kinase surveillance
}

\author{
Magdalena Opalińska ${ }^{1}$ and Chris Meisinger ${ }^{1,2, *}$ \\ ${ }^{1}$ Institut für Biochemie und Molekularbiologie, ZBMZ \\ ${ }^{2}$ BIOSS Centre for Biological Signalling Studies \\ * Corresponding Author: Chris Meisinger, Stefan-Meier-Straße 17; D-79104 Freiburg, Germany; Tel: +49 761203 5287; E-mail: \\ chris.meisinger@biochemie.uni-freiburg.de
}

\begin{abstract}
Despite the simplicity of the yeast Saccharomyces cerevisiae, its basic cellular machinery tremendously mirrors that of higher eukaryotic counterparts. Thus, this unicellular organism turned out to be an invaluable model system to study the countless mechanisms that govern life of the cell. Recently, it has also enabled the deciphering of signalling pathways that control flux of mitochondrial proteins to the organelle according to metabolic requirements. For decades mitochondria were considered autonomous organelles that are only partially incorporated into cellular signalling networks. Consequently, only little has been known about the role of reversible phosphorylation as a meaningful mechanism that orchestrates mitochondrial biology accordingly to cellular needs. Therefore, research in this direction has been vastly neglected. However, findings over the past few years have changed this view and new exciting fields in mitochondrial biology have emerged. Here, we summarize recent discoveries in the yeast model system that point towards a vital role of reversible phosphorylation in regulation of mitochondrial protein import.
\end{abstract}

doi: $10.15698 /$ mic2014.01.127 Received originally: 20.12.2013; Accepted 19.01.2014,

Published 29.01.2014.

Keywords: Mitochondria, organellar biogenesis, preprotein, TOM complex, Casein kinase (CK1), (CK2), Protein kinase $A$ (PKA)

\section{INTRODUCTION}

Mitochondria are double membrane-bound organelles that have evolved from oxygen-scavenging purple eubacteria. The remarkable symbiotic relationship that had started more than 2 billion years ago allowed cells to acquire the ability to survive in a new aerobic environment. Mitochondria play a central role in conversion of energy sustaining viability and cell functions. However, mitochondrial function is not limited to mere ATP production. The organelles have vital roles in numerous cellular processes including metabolism of amino acids and lipids, biosynthesis of heme and iron-sulfur clusters, maintenance of ion concentrations or cell proliferation and death [1-6]. The complexity and indispensability of mitochondrial function in eukaryotes have demanded the coevolution of a variety of sophisticated communication mechanisms between mitochondria and the rest of the cell that utilize ions, reactive oxygen species, metabolites and transcription factors. Emerging evidence points out that reversible phosphorylation likely constitutes a major tool in the regulation of mitochondrial biology and communication [7-9].

Reversible phosphorylation is a ubiquitous posttranslational protein modification. It is estimated that approximately $30 \%$ of cellular proteins are phosphorylated during their lifetime [10]. Covalently bound phosphate groups may influence protein stability, activity or the ability to interact with partner macromolecules. Remarkably, reversible phosphorylation might even influence subcellular localization of the modified protein. The tight interplay between protein kinases and phosphatases determines the level of protein phosphorylation corresponding to the physiological state of the cell. Imbalance in the coordinated action of these opposing enzymes is often associated with human diseases like cancer $[10,11]$.

Mitochondrial activity can be adjusted according to cellular demands at distinct levels e.g. by tuning the ratio between fission and fusion events within the mitochondrial network, by changing the rates of organelle turnover, by adjusting the activities of mitochondrial enzymes or by changes in the complete content of the mitochondrial proteome $[5,6,9]$. A gradual stream of reports implies that reversible phosphorylation plays a major role in all of these areas (reviewed in $[7,8]$ ). Here, we will focus on recent advances in regulation of mitochondrial protein import by cytosolic and mitochondrial protein kinases.

\section{OVERVIEW OF THE MAIN MITOCHONDRIAL PROTEIN IMPORT PATHWAYS}

Mitochondria contain up to 1000 (yeast) and 1500 (human) proteins, of which only a few are encoded by the mitochondrial DNA and synthetized inside the organelle [12-14]. Remarkably, the vast majority (> 99\%) of mitochondrial 
proteins are nuclear-encoded. They are synthesized as precursors on free ribosomes in the cytosol, subsequently imported and sorted to the designated submitochondrial location [15-17]. Most of these precursors enter the organelle via the translocase of the outer membrane (TOM complex) that provides the central protein entry gate (Fig. 1). The TOM complex is formed by the central translocation pore Tom40 that associates with the import receptors Tom20, Tom70, Tom22 and the three small Tom proteins Tom5, Tom6 and Tom7. The small Tom proteins play a role in assembly and stability of the TOM complex. Tom20 preferably recognizes presequence-bearing preproteins, while Tom70 has a preference for binding hydrophobic precursors like the metabolite carriers of the inner membrane $[1,18-20]$. Upon initial recognition by these receptors, precursors are transported to the central import receptor Tom 22 and finally transferred to the Tom 40 channel. Next, the precursors are distributed by further translocation and sorting machineries to the various submitochondrial compartments. Precursors carrying a $\mathrm{N}$-terminal, cleavable presequence are sorted mainly to the matrix or inner membrane. This process is mediated by the presequence translocase of the inner membrane, the TIM23 complex.
Presequences are then typically removed by the matrix processing peptidase MPP $[21,22]$.

Approximately one third of mitochondrial precursors do not contain cleavable presequences and their targeting information is hidden within the mature polypeptides. Precursors of the family of metabolite carriers contain such internal targeting signals. Once released from the TOM complex, these hydrophobic proteins pass through the intermembrane space utilizing the Tim9-Tim10 chaperone complex. Finally, they assemble in the inner membrane in a process driven by the carrier translocase (TIM22 complex) $[15-17,19,20]$.

Some intermembrane space proteins are synthetized as presequence-bearing precursors and employ the TOM and TIM23 complexes to reach their destination. The TIM23 machinery hereby catalyzes a lateral movement of these precursors into the inner membrane followed by cleavage via the inner membrane peptidase IMP that releases the mature protein to the intermembrane space $[21,22]$. Many other intermembrane space proteins utilize an import route that requires the coordinated action of the TOM complex and the specialized import and assembly machinery MIA [15-17, 23-26].

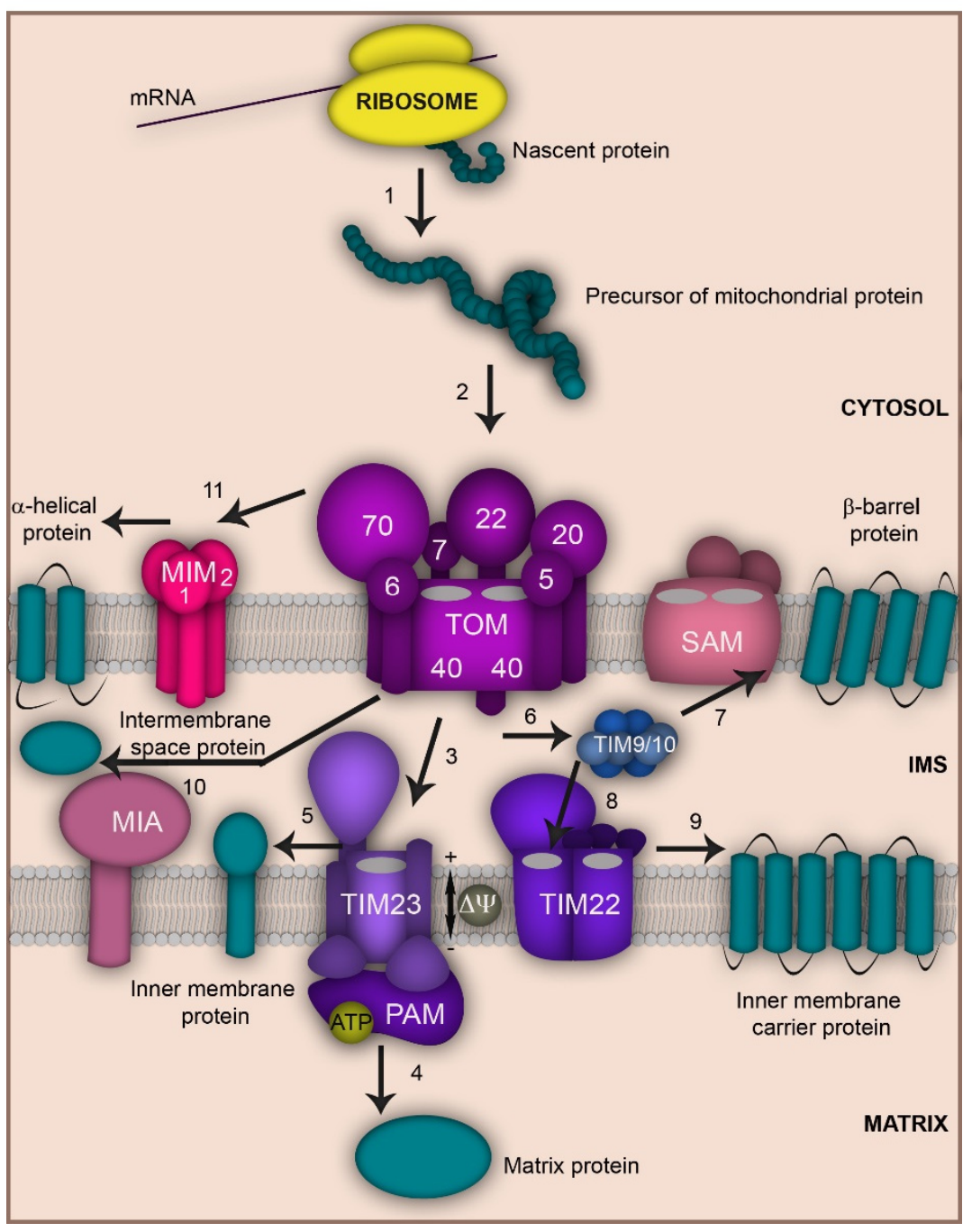

FIGURE 1: Overview of the main mitochondrial protein import pathways. The majority of mitochondrial proteins are synthesized as precursors on free ribosomes in the cytosol (1). Precursors are guided by cytosolic chaperones to the main entry gate of mitochondria, the TOM complex (2). Precursors destined to the matrix pass through the TOM (2) and TIM23 (3) translocases and require the membrane potential $\Delta \psi$. Import into the matrix is facilitated by the Hsp70 containing motor complex PAM that hydrolyzes ATP (4). Precursors of single spanning inner membrane proteins cross the mitochondrial outer membrane through the TOM complex (2) and are laterally released into the mitochondrial inner membrane by the TIM23 machinery (3 and $\mathbf{5}$ ). Precursors of outer membrane $\beta$-barrel proteins also use the TOM complex (2). In the intermembrane space (IMS) they are delivered to the sorting and assembly machinery SAM with the help of small Tim chaperones ( 6 and 7 ) from where they are subsequently inserted into the outer membrane. Precursors of metabolite carriers cross the outer membrane via TOM (2) and are guided by small Tim chaperones (6) to the TIM22 translocase (8) that facilitates their insertion into the inner membrane (9). Many precursors destined to the intermembrane space utilize the TOM complex (2) and the MIA machinery (10). Precursors of $\alpha$-helical outer membrane proteins associate with the Tom70 receptor and are subsequently inserted into the mitochondrial outer membrane and typically depend on the MIM machinery (11). 
Mitochondrial outer membrane proteins comprise both $\beta$-barrel and $\alpha$-helical proteins that are all synthetized in the cytosol and have internal targeting signals. Precursors of $\beta$-barrel proteins are imported across the TOM complex to the intermembrane space, from where they are guided by the Tim9-Tim10 complex to the sorting and assembly machinery (SAM) that mediates their integration into the outer membrane [16-20,27,28]. Coupling of both outer membrane machineries into a TOM-SAM-supercomplex seems to accelerate precursor transfer between the translocases [29]. The insertion of $\alpha$-helical proteins into the outer membrane is less understood. Many precursors of $\alpha$ helical outer membrane proteins seem to be independent of the TOM complex, but require the MIM complex (Mim1containing complex) that consists of Mim1 and Mim2 [20,30-32].

\section{MITOCHONDRIAL PROTEIN IMPORT IS TIGHTLY CONTROLLED BY CYTOSOLIC AND OUTER MEMBRANE BOUND KINASES}

As the TOM complex provides the central entry gate for most nuclear-encoded mitochondrial precursors it constitutes a perfect communication hub for tuning of mitochondrial activity. Indeed, recent studies show that the TOM complex is a major target for regulation of mitochondrial protein biogenesis by cytosolic and outer membrane bound protein kinases [33-35]. Central studies were performed using the unicellular budding yeast Saccharomyces cerevisiae, a versatile model organism to investigate many complex mechanisms that govern life of an eukaryotic cell.

Under glucose-rich conditions, S. cerevisiae predominantly acquires the cellular 'energy currency' ATP in a process called fermentation, in which pyruvate (the end product of glycolysis) is reduced to ethanol and carbon dioxide. Fermentation allows regeneration of $\mathrm{NAD}^{+}$coenzyme pools independently of mitochondrial respiratory activity. While mitochondrial respiratory function is reduced during fermentation, numerous mitochondrial activities that are vital for cell survival have to be preserved (e.g. Fe-S cluster biogenesis [4]). Therefore, cells sense glucose levels and modulate their metabolism for instance by changing the expression or activity of several proteins. The TOM complex hereby constitutes a critical site at which mitochondrial protein import can be adjusted to metabolic switches, e.g. function and biogenesis of the TOM complex are tightly controlled by two kinases involved in glucose-induced signal transduction, CK1 (casein kinase 1) and PKA (cAMPdependent protein kinase) [33-35].

In S. cerevisiae, PKA activity is induced under fermentable conditions. Under non-fermentable conditions, PKA exists in the cytoplasm in its inactive form in which three catalytic subunits (Tpk1, Tpk2, Tpk3) are bound to one regulatory subunit (Bcy1). The presence of glucose induces an increase in CAMP levels that is mediated by the adenylyl cyclase (Cyr1). Subsequent binding of cAMP to the Bcy1 subunit causes dissociation of the PKA complex and release of the catalytic Tpk subunits [36]. In its active form PKA targets Tom70, Tom40 and Tom22, all three critical con- stituents of the TOM complex, and thereby influences mitochondrial protein import capacity on several levels [3335].

On the one hand, PKA negatively regulates import of the channel forming subunit Tom40 (Fig. 2A). It phosphorylates the Tom40 precursor at position Ser54 in the cytosol, prior to its import into the outer membrane. The phosphorylation still allows precursor binding to the outer membrane but prevents its integration into the membrane [34]. Similarly, PKA phosphorylation of the Tom 22 precursor at position Thr76 inhibits its import into mitochondria [35].

On the other hand, regulation of the TOM complex by PKA is not limited to the downregulation of TOM biogenesis. PKA-dependent phosphorylation may also directly modulate the function of the mature TOM complex, reflected by phosphorylation of Tom70 by PKA (Fig. 2B). Tom70, which is the main import receptor for the metabolite carrier family of the inner membrane (e.g. ADP/ATP carrier, phosphate carrier), is phosphorylated at Ser174 upon metabolic switch from respiratory to fermentable conditions [33]. Tom70 interacts with the cytosolic chaperone Hsp70 that delivers hydrophobic precursors to the import receptor [37]. The N-terminal domain of Tom70 recognizes the negatively-charged motif Glu-Glu-Val-Asp (EEVD) at the C-terminus of Hsp70. Introduction of negative charges in the $\mathrm{N}$-terminal domain of Tom70 via phosphorylation at Ser174 significantly affects electrostatic interactions between Tom70 and the precursor-bound chaperone. This leads to an impaired import of metabolic carriers into mitochondria [33]. Thus, PKA modulates mitochondrial activity according to metabolic changes, not only by influencing biogenesis of vital TOM components, but also by compromising Tom70 receptor activity.

In addition to PKA, casein kinase 1 (CK1) is also involved in glucose-driven signalling and influences mitochondrial function in S. cerevisiae (Fig. 2C). CK1 kinase was found to be mainly located at the plasma membrane [38]. A metabolic switch from respiration to fermentation leads to retranslocation from the plasma membrane to the mitochondrial outer membrane [35]. Both subcellular localizations are palmitoylation-dependent and require the activity of the palmitoyl transferase Akr1 $[35,39]$. At mitochondria CK1 specifically modifies Tom 22 at Thr57 supporting its interaction with Tom 20 and assembly into the TOM complex. CK1 supports the assembly of Tom 22 also in the presence of active PKA [35]. This emphasizes the extreme complexity of signalling pathways that are involved in the control of mitochondrial import machineries. While PKA acts on earlier stages of Tom 22 biogenesis, CK1 rather alleviates the inhibitory effects of PKA on the TOM complex than to abolish them. This might be required to maintain crucial mitochondrial functions (e.g. Fe-S cluster synthesis) that are essential under all metabolic conditions. Collectively, these overlapping signalling events ensure accurate metabolic-driven regulation of the function of the main protein entry gate of mitochondria. 


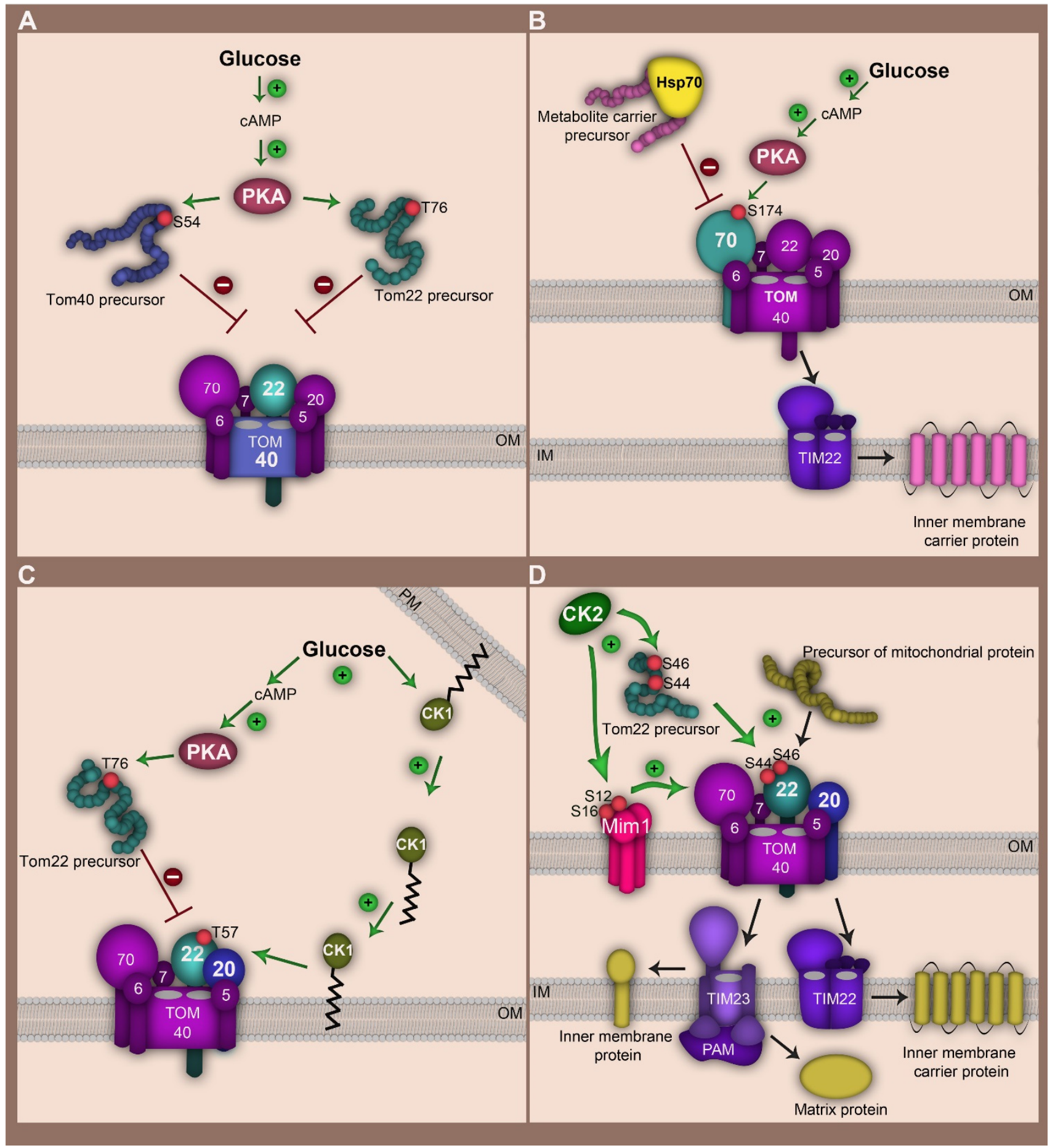

FIGURE 2: Regulation of the TOM complex by cytosolic and outer membrane bound kinases in the yeast S. cerevisiae. (A) Glucose induces an increase in cAMP synthesis and PKA activation. PKA phosphorylates Tom40 and Tom22 precursors in the cytosol at serine 54 and threonine 76, respectively. Translocation of phosphorylated precursors to the outer membrane and their assembly into the mature TOM complex is thereby impaired. (B) Glucose-driven activation of PKA inhibits import of metabolite carrier precursors. PKA phosphorylates the Tom70 receptor at serine 174. This modification impairs interaction of Tom70 with the metabolite carrier/chaperone complex. (C) Glucose signalling leads to retranslocation of CK1 from the plasma membrane (PM) to the mitochondrial outer membrane. CK1 targets threonine 57 at Tom22 promoting Tom22 assembly (by enhancing its interaction with Tom20). CK1 acts downstream of PKA thereby mitigating strong PKA activity. (D) CK2 exerts a stimulatory role on TOM complex biogenesis and activity. Phosphorylation of Tom22 precursor at serine 44 and serine 46 facilitates its interaction with Tom20 receptor and stimulates assembly of Tom20 into the TOM complex. In addition CK2 modifies serine 16 and serine 14 of Mim1 enhancing its stability and promoting Mim1-dependent import of Tom20 and Tom70 precursors. CK2 regulation of the TOM-complex thereby influences downstream import pathways. OM, mitochondrial outer membrane; IM, mitochondrial inner membrane. 
Among the TOM complex subunits 31 residues were identified to be phosphorylated, and many sites emerged to be targets of casein kinase 2 (CK2) [33]. In contrast to PKA, CK2 stimulates mitochondrial protein import by positively regulating biogenesis and function of the TOM complex (Fig. 2D). CK2 seems to be involved in surveillance of many cellular processes including cell proliferation, growth and survival [40-42]. Remarkably, CK2-dependent phosphorylation of Tom22, Tom 20 and Mim1 has strong consequences for mitochondrial protein biogenesis. So far, the central import receptor Tom 22 seems to be the major target of CK2. The CK2-dependent phosphorylations of Tom 22 at Ser44 and Ser46 appear to be constitutively present in the mature TOM. This posttranslational modification occurs already in the cytosol at the early stage of Tom 22 biogenesis. It stimulates interaction of Tom 22 precursor with the import receptors, but phosphorylation of Tom 22 is also required for the assembly of Tom 20 to the mature TOM complex [33]. Phosphorylation of Tom20 by CK2 occurs at Ser172 and seems not to play a role in regulation of its biogenesis or import of other precursor proteins. Still, CK2 controls biogenesis of Tom20 indirectly, by phosphorylation of Tom22 and also Mim1. Mim1 is not a bona fide component of the TOM complex instead it transiently associates with TOM and is vital for the biogenesis of many Tom precursors, including Tom70, Tom20 or the small Tom proteins $[17,31,32]$. CK2 efficiently phosphorylates Mim1 at Ser12 and Ser16. Significant reduction of Mim1 protein levels in conditional $c k 2$ mutants was observed pointing towards a role of CK2 in maintaining Mim1 stability. The mechanistic details of this phenomenon still remain elusive. Due to its role in Mim1 stabilization, phosphorylation of Ser12 and Ser16 exerts a substantial role in efficiency of Mim1-dependent import which influences amongst others Tom20 and Tom70 levels at mitochondria [33]. Taken together, these examples reflect the complexity of signalling pathways engaged in adjustment of mitochondrial activity by directly controlling the mitochondrial proteome at the level of the main translocase of the outer membrane in response to specific cellular demands.

\section{PERSPECTIVES}

The view that mitochondria due to their evolutionary origin are autonomous entities has substantially changed in recent years. Currently, it is evident that the 'power plants of the cell' are not only competent to sense stimuli, but they also actively exchange signals with other cellular compartments. Our current knowledge that reflects the expanding roles of mitochondria in various vital cellular processes that go beyond mere energy conversion underscores the requirement to integrate these organelles with the rest of the cell. Malfunction of mitochondria is linked to many human diseases. Hence, the deciphering of complex signal transduction networks between these organelles and other cellular compartments is of great importance. Reversible phosphorylation is the most widespread signalling mechanism that constitutes the core of cellular communication systems. A gradual stream of reports points out that this mode of posttranslational modification plays also a pivotal role in adjustment of mitochondrial activity. This for instance is illustrated by regulation of mitochondrial protein import by cytosolic and mitochondrial protein kinases. Findings have unveiled complicated networks of overlapping signalling events that target the main protein entry gate of the mitochondrial outer membrane. Given that at least 31 residues of the TOM complex are targets of kinasedependent modifications, and that the significance of only few of them have been unravelled so far, the picture is still far from being complete [33]. The kinome of $S$. cerevisiae consists of at least 130 kinases [43]. It is therefore very probable that the list of players involved in regulation of the mitochondrial protein import machinery will expand in the near future. Interestingly, in vitro screens using purified TOM subunits and numerous recombinantly expressed kinases imply an engagement of additional signalling cascades in regulation of the TOM complex [33]. Besides the TOM complex the outer membrane contains additional membrane protein complexes involved in protein biogenesis (e.g. SAM or ER-mitochondria encounter structure (ER$\mathrm{MES}) /$ mitochondria distribution and morphology protein (MDM) complexes). Furthermore, the existence of TOMSAM supercomplexes has been reported recently. It is therefore tempting to speculate that kinase-phosphatase pairs are involved in the control of biogenesis, activity or supramolecular organisation of these complexes. Moreover the tight regulation of protein import machineries by reversible phosphorylation at inner mitochondrial protein translocases (e.g. TIM complexes or MIA machinery) cannot be excluded and still awaits survey. The discovery of numerous phosphorylated proteins inside mitochondria implies the existence of many signalling cascades that employ kinases and phosphatases in the mitochondrial interior $[7,44]$. Recent developments in the isolation of highly pure mitochondrial fractions combined with mass spectrometry analysis revealed the presence of many kinases and phosphatases in mitochondria (listed in [45]). In S. cerevisiae, most of these signalling proteins have already well-established roles that seem to be performed outside mitochondria. Noteworthy, also kinases and phosphatases with unknown functions have been found in the mitochondrial proteome. Despite the fact that these kinases and phosphatases could represent contamination of mitochondrial fractions by other cellular compartments, these data will provide a starting point to explore the fascinating field of regulation of mitochondrial biology. Furthermore, many signalling molecules could be vastly overlooked using proteomic approaches due to the potentially low abundance of these proteins. In silico analyses predicted that $5 \%$ of the proteins identified in the $S$. cerevisiae kinome are targeted to mitochondria [46]. Future studies will unquestionably lead to the unveiling of further intriguing regulatory mechanisms that govern mitochondrial biology.

\section{ACKNOWLEDGMENTS}

This work was supported by the Deutsche Forschungsgemeinschaft, Excellence Initiative of the German Federal \& 
State Governments (EXC 294 BIOSS) and the Bundesministerium für Bildung und Forschung (Dynamo).

\section{CONFLICT OF INTEREST}

The authors declare no conflict of interest.

\section{COPYRIGHT}

(C) 2014 Opalińska et al. This is an open-access article released under the terms of the Creative Commons Attribution-NonCommercial-NonDerivative 3.0 license, which al-

\section{REFERENCES}

1. Dolezal P, Likic V, Tachezy J, Lithgow T (2006). Evolution of the molecular machines for protein import into mitochondria. Science 313(5785):314-318.

2. Newmeyer DD, Ferguson-Miller S (2003). Mitochondria: releasing power for life and unleashing the machineries of death. Cell 112(4):481-490.

3. Green DR, Kroemer G (2004). The pathophysiology of mitochondrial cell death. Science 305(5684):626-629.

4. Lill R, Mühlenhoff U (2005). Iron-sulfur-protein biogenesis in eukaryotes. Trends Biochem Sci 30(3):133-141.

5. Galluzzi L, Kepp O, Kroemer G (2012). Mitochondria: master regulators of danger signalling. Nat Rev Mol Cell Biol 13(12):780-788.

6. Nunnari J, Suomalainen A (2012). Mitochondria: in sickness and in health. Cell 148(6):1145-1159.

7. Pagliarini DJ, Dixon JE (2006). Mitochondrial modulation: reversible phosphorylation takes center stage? Trends Biochem Sci 31(1):26-34.

8. Kanamaru Y, Sekine S, Ichijo H, Takeda K (2012) The phosphorylation-dependent regulation of mitochondrial proteins in stress responses. J Signal Transduct 2012:931215.

9. McBride HM, Neuspiel M, Wasiak S (2006) Mitochondria: more than just a powerhouse. Curr Biol 16(14):R551-R560.

10. Hunter T (1995). Protein kinases and phosphatases: the yin and yang of protein phosphorylation and signaling. Cell 80(2):225-236.

11. Cohen $P$ (2002). Protein kinases--the major drug targets of the twenty-first century? Nat Rev Drug Discov 1:309-315.

12. Sickmann A, Reinders J, Wagner $Y$, Joppich C, Zahedi R, Meyer HE, Schönfisch B, Perschil I, Chacinska A, Guiard B, Rehling P, Pfanner N, Meisinger $C$ (2003). The proteome of Saccharomyces cerevisiae mitochondria. Proc Natl Acad Sci U S A 100(23):13207-13212.

13. Perocchi $F$, Jensen $\amalg$, Gagneur J, Ahting $U$, von Mering $C$, Bork $P$, Prokisch H, Steinmetz LM (2006). Assessing systems properties of yeast mitochondria through an interaction map of the organelle. PLoS Genet 2(10):e170

14. Pagliarini DJ, Calvo SE, Chang B, Sheth SA, Vafai SB, Ong SE, Walford GA, Sugiana C, Boneh A, Chen WK, Hill DE, Vidal M, Evans JG, Thorburn DR, Carr SA, Mootha VK (2008). A mitochondrial protein compendium elucidates complex I disease biology. Cell 134(1):112123

15. Neupert W, Herrmann JM. (2007). Translocation of proteins into mitochondria. Annu Rev Biochem 76:723-749.

16. Chacinska A, Koehler CM, Milenkovic D, Lithgow T, Pfanner N (2009). Importing mitochondrial proteins: machineries and mechanisms. Cell 138(4):628-644. lows readers to download the article and share it with others, provided that the original authors and source are acknowledged. The article cannot be changed in any way or used commercially.

Please cite this article as: Magdalena Opalińska and Chris Meisinger (2014). Mitochondrial protein import under kinase surveillance. Microbial Cell 1(2): 51-57. doi: 10.15698/mic2014.01.127

17. Schmidt O, Pfanner N, Meisinger C (2010). Mitochondrial protein import: from proteomics to functional mechanisms. Nat Rev Mol Cell Biol 11(9):655-667.

18. Becker T, Vögtle FN, Stojanovski D, Meisinger C (2008). Sorting and assembly of mitochondrial outer membrane proteins. Biochim Biophys Acta 1777(7-8):557-563.

19. Endo T, Yamano K, Kawano S (2011). Structural insight into the mitochondrial protein import system. Biochim Biophys Acta 1808(3):955-970.

20. Dimmer KS, Rapaport D (2012). Unresolved mysteries in the biogenesis of mitochondrial membrane proteins. Biochim Biophys Acta 1818(4):1085-1090.

21. Mossmann D, Meisinger C, Vögtle FN (2012). Processing of mitochondrial presequences. Biochim Biophys Acta 1819(9-10):1098-1106.

22. Teixeira PF, Glaser E (2013). Processing peptidases in mitochondria and chloroplasts. Biochim Biophys Acta 1833(2):360-370.

23. Stojanovski D, Müller JM, Milenkovic D, Guiard B, Pfanner N, Chacinska $A$ (2008). The MIA system for protein import into the mitochondrial intermembrane space. Biochim Biophys Acta 1783(4):610617.

24. Endo T, Yamano K, Kawano S (2010). Structural basis for the disulfide relay system in the mitochondrial intermembrane space. Antioxid Redox Signal 13(9):1359-1373.

25. Sideris DP, Tokatlidis K (2010). Oxidative protein folding in the mitochondrial intermembrane space. Antioxid Redox Signal 13(8):1189-11204.

26. Herrmann JM, Riemer J (2012). Mitochondrial disulfide relay: redox-regulated protein import into the intermembrane space. J Biol Chem 287(7):4426-4433.

27. Kutik S, Stojanovski D, Becker L, Becker T, Meinecke M, Krüger V, Prinz C, Meisinger C, Guiard B, Wagner R, Pfanner N, Wiedemann N (2008). Dissecting membrane insertion of mitochondrial beta-barrel proteins. Cell 132(6):1011-1024.

28. Paschen SA, Neupert W, Rapaport D (2005). Biogenesis of betabarrel membrane proteins of mitochondria. Trends Biochem Sci 30(10):575-582.

29. Qiu J, Wenz LS, Zerbes RM, Oeljeklaus S, Bohnert M, Stroud DA, Wirth C, Ellenrieder L, Thornton N, Kutik S, Wiese S, Schulze-Specking A, Zufall N, Chacinska A, Guiard B, Hunte C, Warscheid B, van der Laan M, Pfanner N, Wiedemann N, Becker T (2013). Coupling of mitochondrial import and export translocases by receptor-mediated supercomplex formation. Cell 154(3):596-608.

30. Dimmer KS, Papić D, Schumann B, Sperl D, Krumpe K, Walther DM, Rapaport D (2012). A crucial role for Mim2 in the biogenesis of mitochondrial outer membrane proteins. J Cell Sci 125(Pt 14):3464-3473. 
31. Becker T, Pfannschmidt S, Guiard B, Stojanovski D, Milenkovic D, Kutik S, Pfanner N, Meisinger C, Wiedemann N (2008). Biogenesis of the mitochondrial TOM complex: Mim1 promotes insertion and assembly of signal-anchored receptors. J Biol Chem 283(1):120-127.

32. Becker T, Wenz LS, Krüger V, Lehmann W, Müller JM, Goroncy L, Zufall N, Lithgow T, Guiard B, Chacinska A, Wagner R, Meisinger C, Pfanner $N$ (2011). The mitochondrial import protein Mim1 promotes biogenesis of multispanning outer membrane proteins. J Cell Biol 194(3):387-395.

33. Schmidt O, Harbauer AB, Rao S, Eyrich B, Zahedi RP, Stojanovski D, Schönfisch B, Guiard B, Sickmann A, Pfanner N, Meisinger C (2011). Regulation of mitochondrial protein import by cytosolic kinases. Cell 144(2):227-239.

34. Rao S, Schmidt O, Harbauer AB, Schönfisch B, Guiard B, Pfanner N, Meisinger $C$ (2012). Biogenesis of the preprotein translocase of the outer mitochondrial membrane: protein kinase A phosphorylates the precursor of Tom40 and impairs its import. Mol Biol Cell 23(9):16181627.

35. Gerbeth C, Schmidt O, Rao S, Harbauer AB, Mikropoulou D, Opalińska M, Guiard B, Pfanner N, Meisinger C (2013). Glucoseinduced regulation of protein import receptor Tom 22 by cytosolic and mitochondria-bound kinases. Cell Metab 18(4):578-587.

36. Zaman S, Lippman SI, Zhao X, Broach JR (2008). How Saccharomyces responds to nutrients. Annu Rev Genet 42:27-81.

37. Young JC, Hoogenraad NJ, Hartl FU (2003). Molecular chaperones Hsp90 and Hsp70 deliver preproteins to the mitochondrial import receptor Tom70. Cell 112(1):41-50.

38. Vancura A, Sessler A, Leichus B, Kuret J (1994). A prenylation motif is required for plasma membrane localization and biochemical function of casein kinase I in budding yeast. J Biol Chem 269(30):1927119278
39. Feng Y, Davis NG (2000). Akr1p and the type I casein kinases act prior to the ubiquitination step of yeast endocytosis: Akr1p is required for kinase localization to the plasma membrane. Mol Cell Biol 20(14):5350-5359.

40. Olsten ME, Litchfield DW (2005). Order or chaos? An evaluation of the regulation of protein kinase CK2. Biochem Cell Biol 82(6):681-693.

41. Pinna LA, Allende JE (2009). Protein kinase CK2 in health and disease: Protein kinase CK2: an ugly duckling in the kinome pond. Cell Mol Life Sci 66(11-12):1795-1799.

42. Poole A, Poore T, Bandhakavi S, McCann RO, Hanna DE, Glover CV (2005). A global view of CK2 function and regulation. Mol Cell Biochem 274(1-2):163-170.

43. Breitkreutz A, Choi H, Sharom JR, Boucher L, Neduva V, Larsen B, Lin ZY, Breitkreutz BJ, Stark C, Liu G, Ahn J, Dewar-Darch D, Reguly T, Tang X, Almeida R, Qin ZS, Pawson T, Gingras AC, Nesvizhskii Al, Tyers $M$ (2010). A global protein kinase and phosphatase interaction network in yeast. Science 328(5981):1043-1046.

44. Reinders J, Wagner K, Zahedi RP, Stojanovski D, Eyrich B, van der Laan M, Rehling P, Sickmann A, Pfanner N, Meisinger C (2007). Profiling phosphoproteins of yeast mitochondria reveals a role of phosphorylation in assembly of the ATP synthase. Mol Cell Proteomics 2007 6(11):1896-1906.

45. Rao S, Gerbeth C, Harbauer A, Mikropoulou D, Meisinger C, Schmidt $O$ (2011). Signaling at the gate: phosphorylation of the mitochondrial protein import machinery. Cell Cycle 10(13):2083-2090.

46. Tomaska $L$ (2000). Mitochondrial protein phosphorylation: lessons from yeasts. Gene 255(1):59-64. 